\title{
Percutaneous repair of sinus venosus ASD: the end of congenital cardiac surgery?
}

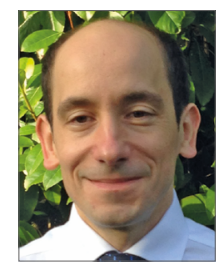

Aleksander Kempny ${ }^{1,2 *}$, MD, PhD; Michael A. Gatzoulis ${ }^{1,2}, \mathrm{MD}, \mathrm{PhD}$, FACC

1. Adult Congenital Heart Centre and National Centre for Pulmonary Hypertension, Royal Brompton Hospital, London, United Kingdom; 2. National Heart and Lung Institute, Imperial College School of Medicine, London, United Kingdom

Atrial septal defect (ASD) is one of the most common congenital heart defects with a prevalence of isolated defect ranging from 0.5 to 2.5 cases per 1,000 live births ${ }^{1}$. The direction and volume of the resulting shunt depend on the size of the defect, its type, and on the pressures in the right and left atrium. These, in turn, depend on the function of the left and right atrioventricular valve and on the diastolic properties of both ventricles. In most patients with isolated ASD and without pulmonary hypertension $(\mathrm{PH})$, there is a left to right shunt. Over time, the additional volume load of the right ventricle (RV) resulting from shunting causes RV dilatation, which is a prerequisite for advocating closure. In ASD patients with a left to right shunt, but without obvious RV dilatation on echocardiography, further shunt quantification may be performed using cardiac catheterisation or cardiac magnetic resonance imaging (MRI). Closure of defects with signs of significant shunt, resulting in right heart dilation or documented significantly increased transpulmonary to systemic blood flow ratio ( $\mathrm{Qp} / \mathrm{Qs}>1.5)$, is associated with improved clinical outcomes as compared to conservative therapy, regardless of age ${ }^{2}$.

There are multiple factors supporting early ASD closure. Right heart dilation is associated with increased risk of arrhythmia, progressive tricuspid regurgitation and right heart failure. Patients may present at diagnosis with few or no obvious symptoms. This may, however, result from chronic adaptation to abnormal haemodynamics; objective assessment of exercise performance with cardiopulmonary exercise testing often demonstrates impaired exercise capacity. Moreover, a subset of patients may develop pulmonary hypertension. In a few, this may result in reversal of the shunt and Eisenmenger syndrome physiology.

In the Bethesda classification of congenital heart disease lesions, repaired ASD is listed as a simple defect. However, historic data on natural history clearly demonstrate that ASD is not a benign lesion and, if not corrected, is associated with high mortality. In his series of original publications in the 1960s and 1970s, Dr Maurice Campbell, from Guy's Hospital in London, described the natural history of several congenital cardiac lesions, including ASD. There was a mortality of $12 \%$ at the age of 20 years and $76 \%$ at the age of 50 years, compared to $2 \%$ and $8 \%$ in the normal general population at the time, respectively ${ }^{3}$. One can easily argue that patients diagnosed with an ASD at that time were then at the more severe end of the spectrum and thus more likely to have large shunts and be symptomatic. These historic data are, however, in sharp contrast with contemporary mortality statistics in patients with repaired 
ASD, demonstrating improved if not normalised survival ${ }^{4-7}$. Only a small proportion of patients with ASD may develop pulmonary hypertension later in life and is not suitable for ASD closure. Even when accounting for these patients, contemporary mortality in ASD patients is low. Thus, the difference between contemporary survival data on ASD patients and historic data on its natural history is staggering and strongly in favour of early ASD closure for prognostic benefit. The risk of supraventricular arrhythmia and heart failure increases with age at repair, in keeping with incomplete right atrial and ventricular remodelling in older patients after late ASD closure. Thus, early diagnosis and intervention are essential in reducing associated morbidity ${ }^{8}$.

Successful surgical ASD closure was reported as early as in the 1940 s, with various surgical techniques being developed in the 1950 s and 1960 s, with the advent of cardiopulmonary bypass ${ }^{9}$. The first-in-man reported percutaneous closure of a secundum ASD was performed in 1975 in New Orleans, LA, USA, with a double umbrella, non-retrievable device without self-centring ${ }^{10}$. There has been a dynamic development of various concepts of percutaneous ASD closure since, with double umbrella, self-centring devices currently being the most commonly used. Percutaneous closure offers several advantages as compared to surgical closure. It requires minimal hospital stay, is associated with lower costs and lower utilisation of resources, speedier recovery and, not surprisingly, is preferred by patients. The first successful percutaneous repair of a superior sinus venosus defect was reported by Garg et al in 2014 and has since been described in several case reports from other centres ${ }^{11}$. In the current issue of EuroIntervention, Riahi et al describe a series of consecutive patients diagnosed with superior sinus venosus defect, all managed with a novel percutaneous technique at a single centre ${ }^{12}$.

\section{Article, see page $\mathbf{8 6 8}$}

The authors should be congratulated for achieving excellent midterm outcome, without residual shunt and with preserved patency of the right upper pulmonary vein. There were no major complications. Has another bastion of cardiac surgery therefore been conquered? For the time being, the preferred management of superior sinus venosus ASD in most congenital heart disease centres is likely to remain surgical, for several reasons. The technique used by Riahi et al is more complex and demanding than the one used for percutaneous closure of secundum $\mathrm{ASD}^{12}$. It requires a dedicated team with an interventional cardiologist, a radiologist experienced in performing segmentation for $3 \mathrm{D}$ printing and an advanced, in-house $3 \mathrm{D}$ printer or an established link with a $3 \mathrm{D}$ printing company, certified for the processing of medical data. The prevalence of sinus venosus defect is relatively low and may not justify the investment in personnel and infrastructure, even at large congenital heart disease centres. Moreover, excellence in any procedure requires a sufficient annual volume of cases. Guidelines for percutaneous coronary interventions, for example, advocate a minimum of 50 interventions per year ${ }^{13}$. Whilst this number may be too high for percutaneous closure of superior sinus venosus ASD, considering the low prevalence of this defect, it becomes obvious that a large referral base is required. This requires referring patients, regardless of geographical boundaries, to centres with special interest and confirmed excellence in rare or complex procedures (Figure 1). In fact, this may be a prerequisite

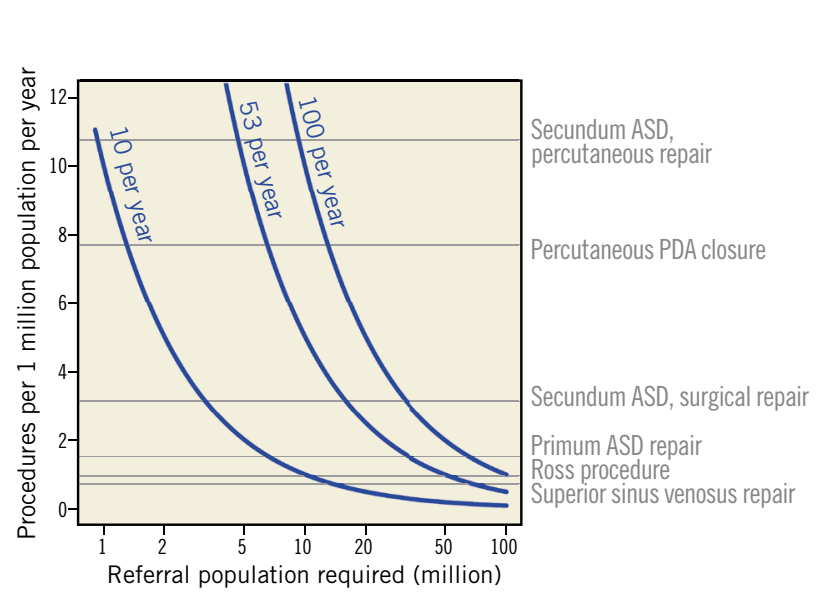

Figure 1. Relationship of referral population required to achieve sufficient case volume for selected procedures. Each of the isolines (blue) represents the relationship of the referral population required (horizontal axis, logarithmic scale) to achieve a certain case volume per year (corresponding blue labels), depending on the frequency of the procedure performed (vertical axis). For example, to achieve a yearly volume of 50 percutaneous closures of secundum atrial septal defect (ASD), one would require a referral population of 6 million. To achieve the same volume of annual cases for superior sinus venosus ASD repair, a referral population of 73 million would be required. As some of these patients may require additional procedures, such as tricuspid repair or coronary artery bypass grafting, the referral population to achieve a sufficient case load for percutaneous closure of superior sinus venosus ASD may be even higher. This analysis highlights the need for national and international referral patterns for rare or complex procedures. Number of procedures performed per 10 million population based on the 2015 data of the National Institute for Cardiovascular Outcomes Research in the United Kingdom; UK population for 2015 as reported by the World Data Bank. Calculations based on an assumed proportion of secundum ASDs of $80 \%$ and $4 \%$ for superior sinus venosus ASD. Percutaneous closure of patent ductus arteriosus (PDA) and the Ross procedure were selected to put the analysis in the context of other procedures. 
for offering rare and complex interventions, requiring advanced expertise of a team. This is where collaboration comes into play with agreed and established referral patterns so that more patients may benefit from novel procedures, such as the one described by Riahi et al, once such procedures have been externally validated ${ }^{12}$.

\section{Conflict of interest statement}

A. Kempny and M. Gatzoulis have in the past received educational or research grants from Bayer UK, GSK, Pfizer UK, Actelion UK and Actelion Global.

\section{References}

1. van der Linde D, Konings EE, Slager MA, Witsenburg M, Helbing WA, Takkenberg JJ, Roos-Hesselink JW. Birth prevalence of congenital heart disease worldwide: a systematic review and meta-analysis. J Am Coll Cardiol. 2011;58:2241-7.

2. Attie F, Rosas M, Granados N, Zabal C, Buendía A, Calderón J. Surgical treatment for secundum atrial septal defects in patients $>40$ years old. A randomized clinical trial. J Am Coll Cardiol. 2001;38:2035-42.

3. Campbell M. Natural history of cyanotic malformations and comparison of all common cardiac malformations. Br Heart $J$. 1972;34:3-8.

4. Gatzoulis MA, Redington AN, Somerville J, Shore DF. Should atrial septal defects in adults be closed? Ann Thorac Surg. 1996;61:657-9.

5. HelberU, Baumann R, SeboldtH, Reinhard U, Hoffmeister HM. Atrial septal defect in adults: cardiopulmonary exercise capacity before and 4 months and 10 years after defect closure. J Am Coll Cardiol. 1997;29:1345-50.

6. Roos-Hesselink JW, Meijboom FJ, Spitaels SE, van Domburg R, van Rijen EH, Utens EM, Bogers AJ, Simoons ML.
Excellent survival and low incidence of arrhythmias, stroke and heart failure long-term after surgical ASD closure at young age. A prospective follow-up study of 21-33 years. Eur Heart J. 2003; 24:190-7.

7. Konstantinides S, Geibel A, Olschewski M, Gornandt L, Roskamm H, Spillner G, Just H, Kasper W. A comparison of surgical and medical therapy for atrial septal defect in adults. $N$ Engl $J$ Med. 1995;333:469-73.

8. Gatzoulis MA, Freeman MA, Siu SC, Webb GD, Harris L. Atrial arrhythmia after surgical closure of atrial septal defects in adults. N Engl J Med. 1999;340:839-46.

9. Alexi-Meskishvili VV, Konstantinov IE. Surgery for atrial septal defect: from the first experiments to clinical practice. Ann Thorac Surg. 2003;76:322-7.

10. Nassif M, Abdelghani M, Bouma BJ, Straver B, Blom NA, Koch KT, Tijssen JG, Mulder BJ, de Winter RJ. Historical developments of atrial septal defect closure devices: what we learn from the past. Expert Rev Med Devices. 2016;13:555-68.

11. Garg G, Tyagi H, Radha AS. Transcatheter closure of sinus venosus atrial septal defect with anomalous drainage of right upper pulmonary vein into superior vena cava--an innovative technique. Catheter Cardiovasc Interv. 2014;84:473-7.

12. Riahi M, Velasco Forte MN, Byrne N, Hermuzi A, Jones M, Baruteau AE, Valverde I, Qureshi SA, Rosenthal E. Early experience of transcatheter correction of superior sinus venosus atrial septal defect with partial anomalous pulmonary venous drainage. EuroIntervention. 2018;14:868-76.

13. Banning AP, Baumbach A, Blackman D, Curzen N, Devadathan S, Fraser D, Ludman P, Norell M, Muir D, Nolan J, Redwood S; British Cardiovascular Intervention society. Percutaneous coronary intervention in the UK: recommendations for good practice 2015. Heart. 2015;101 Suppl 3:1-13. 